\title{
miR-29 promotes osteosarcoma cell proliferation and migration by targeting PTEN
}

\author{
QIULIANG LIU, PEISHUO GENG, LONGYAN SHI, QI WANG and PENGLIANG WANG \\ Department of Pediatric Surgery, The First Affiliated Hospital of Zhengzhou University, \\ Zhengzhou, Henan 450052, P.R. China
}

Received February 6, 2018; Accepted October 10, 2018

DOI: $10.3892 / \mathrm{ol} .2018 .9646$

\begin{abstract}
Osteosarcoma (OS) is an aggressive malignant neoplasm that arises from primitively transformed cells of mesenchymal origin, and that exhibits osteoblastic differentiation and produces malignant osteoid. MicroRNAs (miRNAs) have been widely reported to have important regulatory roles in various human tumors, including OS. However, the potential mechanism of miR-29 in OS remains largely unknown. miR-29 was highly expressed in OS and overexpression of miR-29 promoted OS cell proliferation, as well as proliferating cell nuclear antigen (PCNA) expression and migration, whereas lower expression of miR-29 inhibited OS cell proliferation, PCNA expression and migration. In the present study, a dual-luciferase reporter system supporting phosphatase and tensin homolog (PTEN) was a target of miR-29 and its expression was inhibited by miR-29 mimic, but increased by miR-29 inhibitor. Overexpression of PTEN inhibited OS cell proliferation and migration and it could attenuate miR-29 promotion effect on OS progression. Overall, the results revealed that miR-29, as a tumor promoter, is involved in OS progression and metastasis by targeting PTEN, indicating that the miR-29/PTEN pathway is a potential therapeutic target for the treatment of OS.
\end{abstract}

\section{Introduction}

Osteosarcoma (OS) is a malignant bone tumor characterized by tumor cells directly forming bone or bone tissues. It often occurs in adolescents or children under the age of 20 and the prognosis is poor (1-3). The current treatment strategy focuses on primary tumors, but it is limited in the treatment of metastatic OS (4). Thus, treating metastatic OS is now a great challenge for oncologists. It is necessary for us to understand the pathogenesis and biological characteristics of metastatic

Correspondence to: Dr Qiuliang Liu, Department of Pediatric Surgery, The First Affiliated Hospital of Zhengzhou University, 1 Jianshe East Road, Zhengzhou, Henan 450052, P.R. China E-mail: qn7qkr@163.com

Key words: osteosarcoma, miR-29, PTEN, proliferation, migration
OS and to find new targets and biomarkers for the treatment of OS patients is urgent $(5,6)$.

It is known that, miRNAs can extensively disrupt various functions of different cancers by targeting mRNA (7), and the target mRNA is also proven to regulate cell proliferation, invasion and apoptosis (8). New evidence suggests that miRNAs are involved in human carcinogenesis, including promoting or inhibiting tumor development $(9,10)$. For example, miR-365, miR-124a, miR-625 and miR-141 acted as tumor suppressors in OS (11-14), while miR-504, miR-19 and miR-93 promoted OS cell progression (15-17).

The miR-29 family contains three mature members (miR-29a, miR-29b and miR-29c). An increasing number of studies have shown the decreased expression of miR-29 family in various types of cancer and that it has the ability to suppress tumors, including breast, bladder and pancreatic cancer (18-20). Shin et al found that miR-29b effect on glioblastoma was suppressive (21). Ma et al stated that, miR-29 suppressed schwannoma cell proliferation and motility by regulating CDK6 (22). In colon cancer, miR-29 showed inhibitory effect on cell invasion and migration by regulating MMP2 (23). Additionally, miR-29 suppressed lung cancer development by targeting DNMT3A and DNMT3B (24). However, role of miR-29 in OS and its mechanism have rarely been reported.

It is well known that phosphatase and tensin homolog (PTEN) acts as a tumor suppressor and PTEN expression was proved abnormal in many cancers (25). For instance, PTEN functioned as a tumor inhibitor in regulating pancreatic cancer progression regulated by miR-32 (26). miR-21 modulated gastric cancer development via targeting PTEN (27) and PTEN was also a target of miR-20b in modulating prostate cancer cell viability and migratory ability (28). However, the effect of PTEN on OS cell migration and proliferation and whether PTEN is regulated by miR-29 in OS have yet to be reported.

In our study, miR-29 and PTEN in OS and its potential mechanism in modulating OS cell migration and proliferation was investigated. Our results may provide important insight into the prognosis and treatment of OS.

\section{Materials and methods}

OS specimens. We collected conventional OS and adjacent non-cancerous tissues from 60 patients who underwent resection surgery in the First Affiliated Hospital of Zhengzhou 
University (Zhengzhou, China) between February 2010 and August 2016. None of OS patients had received radiotherapy or chemotherapy before surgery and the tissues were diagnosed as OS by pathologists. All tissue specimens were placed into liquid nitrogen immediately and stored at $-80^{\circ} \mathrm{C}$ in a refrigerator for use in subsequent experiments. The Ethics Committee of Zhengzhou University approved this study and the patients signed informed consent prior to surgery.

Cell lines and cell culture. The purchased OS cell lines (MG-63, U2OS, 143B, Saos-2) were cultured in RPMI-1640 medium at $37^{\circ} \mathrm{C}$ and the medium contained $20 \%$ FBS and penicillin $(100 \mathrm{U} / \mathrm{ml})$ and streptomycin $(100 \mu \mathrm{g} / \mathrm{ml}) . \mathrm{hFOB} 1.19$ (normal human osteoblast) cells were cultured in DMEM supplemented with $10 \% \mathrm{FBS}$ and G418 $(0.03 \mathrm{mg} / \mathrm{ml})$ at $34^{\circ} \mathrm{C}$ with 5\% $\mathrm{CO}_{2}$. OS cell lines (MG-63, U2OS, 143B, Saos-2) and hFOB1.19 (normal human osteoblast) cells were obtained from ATCC (Manassas, VA, USA).

Cell transfection. miR-29 mimic or inhibitor provided by Suzhou GenePharma Co.,Ltd. (Suzhou, China) was transfected into MG-63 cells to facilitate or inhibit miR-29 expression and control mimic were used as control (con). We added MG-63 cells into 24-well plates containing medium and we performed transfection using Lipofectamine 3000 reagent (Invitrogen; Thermo Fisher Scientific, Inc., Waltham, MA, USA) for $48 \mathrm{~h}$. PTEN vector and con vector were synthesized by GenePharma Co., Ltd. and they were used to enforce PTEN expression and acted as a control separately.

RNA extraction and reverse transcription-quantitative polymerase chain reaction ( $R T-q P C R)$. Total RNA and microRNA were extracted from OS tissues and cells using TRIzol reagent (Invitrogen; Thermo Fisher Scientific, Inc.) according to the manufacturer's protocol. Complementary DNA was synthesized using PrimeScript RT reagent kit and RT-qPCR was performed using SYBR Premix Ex Taq (both from Takara Biotechnology Co., Ltd., Dalian, China) with the Stratagene Mx3000P real-time PCR system (Agilent Technologies, Inc., Santa Clara, CA, USA). The primer sequences used were: miR-29-F: TGCCAGGAGCTGGTGATTTCCT, miR-29-R: ACGGGCGTACAGAGGATCCCC. PTEN-F: GTGCAG ATAATGACAAG, PTEN-R: GATTTGACGGCTCCTCT. proliferating cell nuclear antigen (PCNA)-F: GGTGTTG GAGGCACTCAAGG, PCNA-R: CAGGGTGAGCTGCACC AAAG. U6-F: CTCGCTTCGGCAGCAC, U6-R: ACGCTTC ACGAATTTGC. $\beta$-actin-F: GATCATTGCTCCTCCTGAGC; $\beta$-actin-R: ACTCCTGCTTGCTGATCCAC. U6 and $\beta$-actin were used as internal controls. Relative expression of miR-29, PTEN and PCNA was calculated by the $2^{-\Delta \Delta C q}$ method (29).

Western blot analysis. Lysis buffer containing protease inhibitor and phenylmethanesulfonyl fluoride (PMSF) was added into OS tissues or cells and then homogenized on ice (the written informed consent from patients were obtained). After centrifugation at $12,000 \mathrm{x} \mathrm{g}$, at $4^{\circ} \mathrm{C}$ for $30 \mathrm{~min}$, the supernatant was measured by BCA kit. Then, $50 \mu \mathrm{g}$ protein specimens at the same concentration were added onto sodium dodecyl sulfate-polyacrylamide gel electrophoresis (SDS-PAGE). Proteins were then transferred to polyvinylidene fluoride
(PVDF) membranes and blocked with skim milk (5-10\%) at room temperature for $2 \mathrm{~h}$ subsequently. Then primary antibodies, monoclonal rabbit anti-PTEN (1:1,000, cat. no. 9188; Cell Signaling Technology, Inc., Danvers, MA, USA), monoclonal mouse anti-actin (1:1,000, cat. no. sc-58673; Santa Cruz Biotechnology, Inc., Dallas, TX, USA) were added to incubate the membranes at $4^{\circ} \mathrm{C}$ overnight and secondary antibodies, monoclonal goat anti-rabbit or anti-mouse IgG-HRP $(1: 2,000$, cat. no. sc-2004; Santa Cruz Biotechnology, Inc.) were later added to incubate the membranes at room temperature $\left(25^{\circ} \mathrm{C}\right)$ for $2 \mathrm{~h}$. Finally, the enhanced chemiluminescence kit (ECL; Merck Millipore, Billerica, MA, USA) was used to detect the signals. The relative expression of target proteins was evaluated using target protein $/ \beta$-actin.

Transwell assay. Transwell assay was used to measure cell migration. Transwell inserts with $8 \mu \mathrm{m}$ pore size polycarbonic membrane (Corning Costar Corp., Cambridge, MA, USA) were used to divide Transwell chamber into upper and lower chambers. MG-63 cells (5x105/well) with different transfection were placed into the top chambers and DMEM containing $10 \%$ FBS into lower chambers and then cultured for $48 \mathrm{~h}$ at $37^{\circ} \mathrm{C}$ under $\mathrm{CO}_{2}$ atmosphere. The cells from the top chambers migrated into the lower chambers, and the cells on the top chambers were removed with cotton. The cells were fixed on lower chambers using $4 \%$ formaldehyde for $30 \mathrm{~min}$ at room temperature $\left(25^{\circ} \mathrm{C}\right)$, cells were stained with $0.1 \%$ crystal violet for $15 \mathrm{~min}$, and images of the cells were captured using an inverted microscope (Nikon 80i, Feasterville, PA, USA). Cells were then counted using Living Image Version 4.5 Software (PerkinElmer, Inc., Waltham, MA, USA) image software.

MTT assay. Fresh culture $(100 \mu 1)$ medium was added into 96-well plates to replace the culture medium when the MG-63 cell population reached optimal densities. Then, MTT reagent $(20 \mu \mathrm{l})$ was added and cultured at $37^{\circ} \mathrm{C}$ for $4 \mathrm{~h}$. Subsequently, the MTT medium was sucked out and $100 \mu \mathrm{l}$ of DMSO was added for additional $10 \mathrm{~min}$, the plates were read at a wavelength of $570 \mathrm{~nm}$ using the multilabel plate reader (PerkinElmer, Inc.) at 1, 2, 3, 4 days to measure the absorbance of each well.

Luciferase reporter assay. TargetScan Human 7.1 was firstly carried out to identify the possible targets of miR-29. We used Lipofectamine 2000 (Thermo Fisher Scientific, Inc.) to co-transfect miR-29 mimic or control mimic with 3'-UTR of wild or mutated PTEN in MG-63 cells. The dual luciferase reporter system (GeneCopoeia, Inc., Rockville, MD, USA) was then used to measure the luciferase activity of MG-63 cells treated with different transfection.

Statistical analysis. All experiments were repeated three times independently. SPSS v.19.0 software (IBM Corp., Armonk, NY, USA) was used to perform statistical analyses and GraphPad Prism 5.02 software (GraphPad Software, Inc., La Jolla, CA, USA) to complete graph presentations. Results are presented as the mean \pm standard deviation, and the data were evaluated using Student's t-test or Tukey's post hoc test after ANOVA in SPSS. P $<0.05$ was considered to indicate a statistically significant difference. 

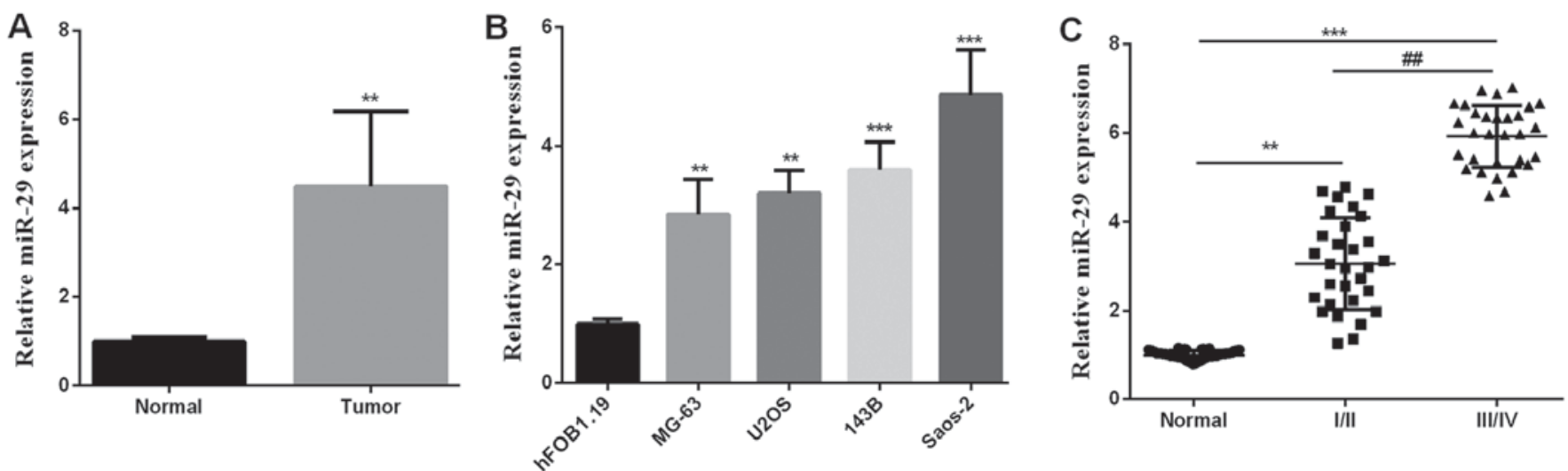

Figure 1. miR-29 is increased in OS. (A) Quantification of miR-29 average expression in OS tissues ( $\mathrm{n}=60$ ). (B) miR-29 measured in OS cells and normal cells. (C) Quantification of miR-29 expression in different stages of OS. ${ }^{* *} \mathrm{P}<0.01,{ }^{* * *} \mathrm{P}<0.001 ;{ }^{\# \#} \mathrm{P}<0.01$. OS, osteosarcoma.

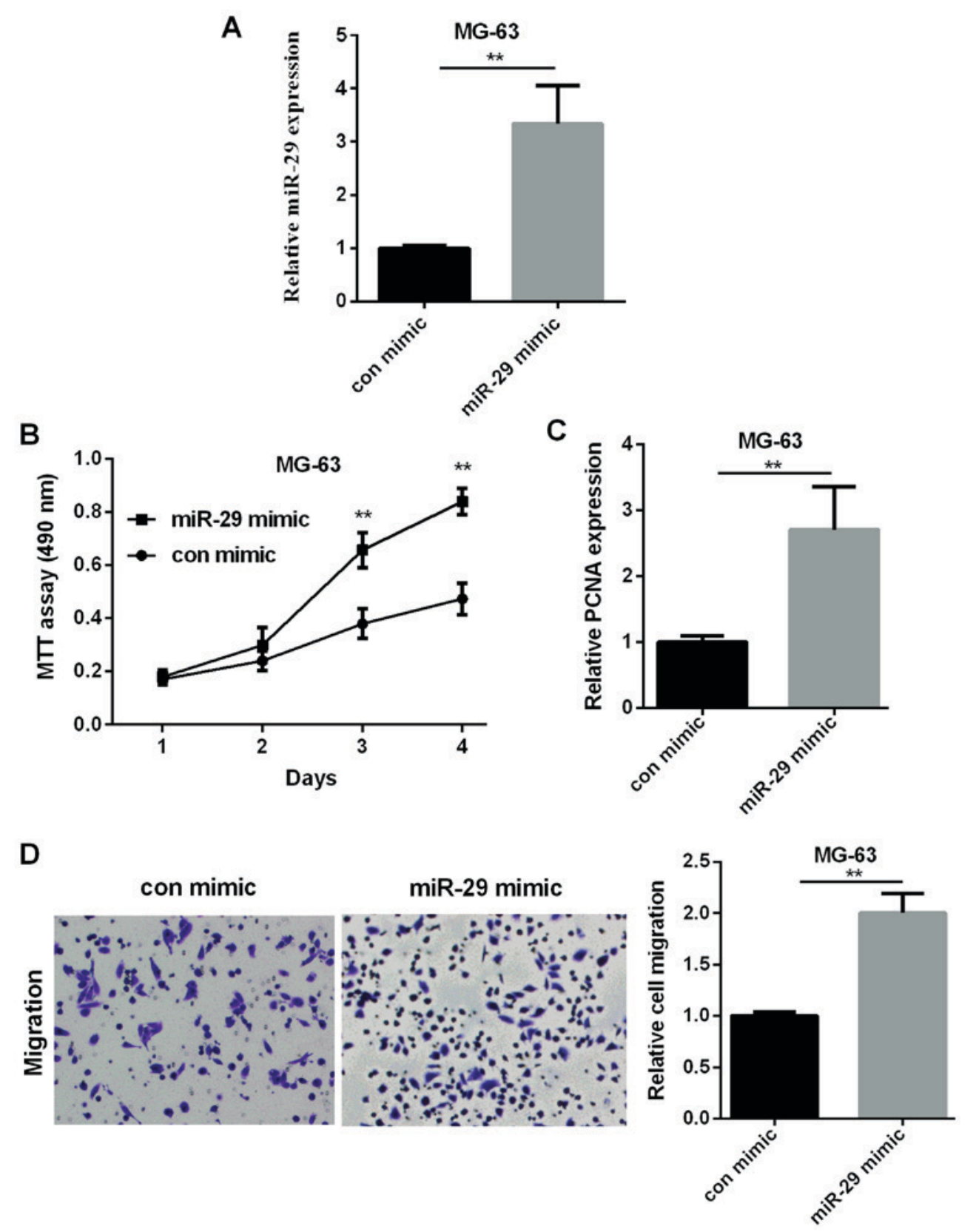

Figure 2. miR-29 mimic promoted OS cell migration and proliferation. (A) Quantification of miR-29 mRNA and protein level in MG-63 cells treated with miR-29 mimic. (B and C) Detection of cell viability by MTT and PCNA expression using RT-qPCR after MG-63 cells restoration of miR-29. (D) Measurement of cell migration by Transwell after MG-63 cell restoration of miR-29. ${ }^{* *} \mathrm{P}<0.01$. OS, osteosarcoma; con, control. 
A
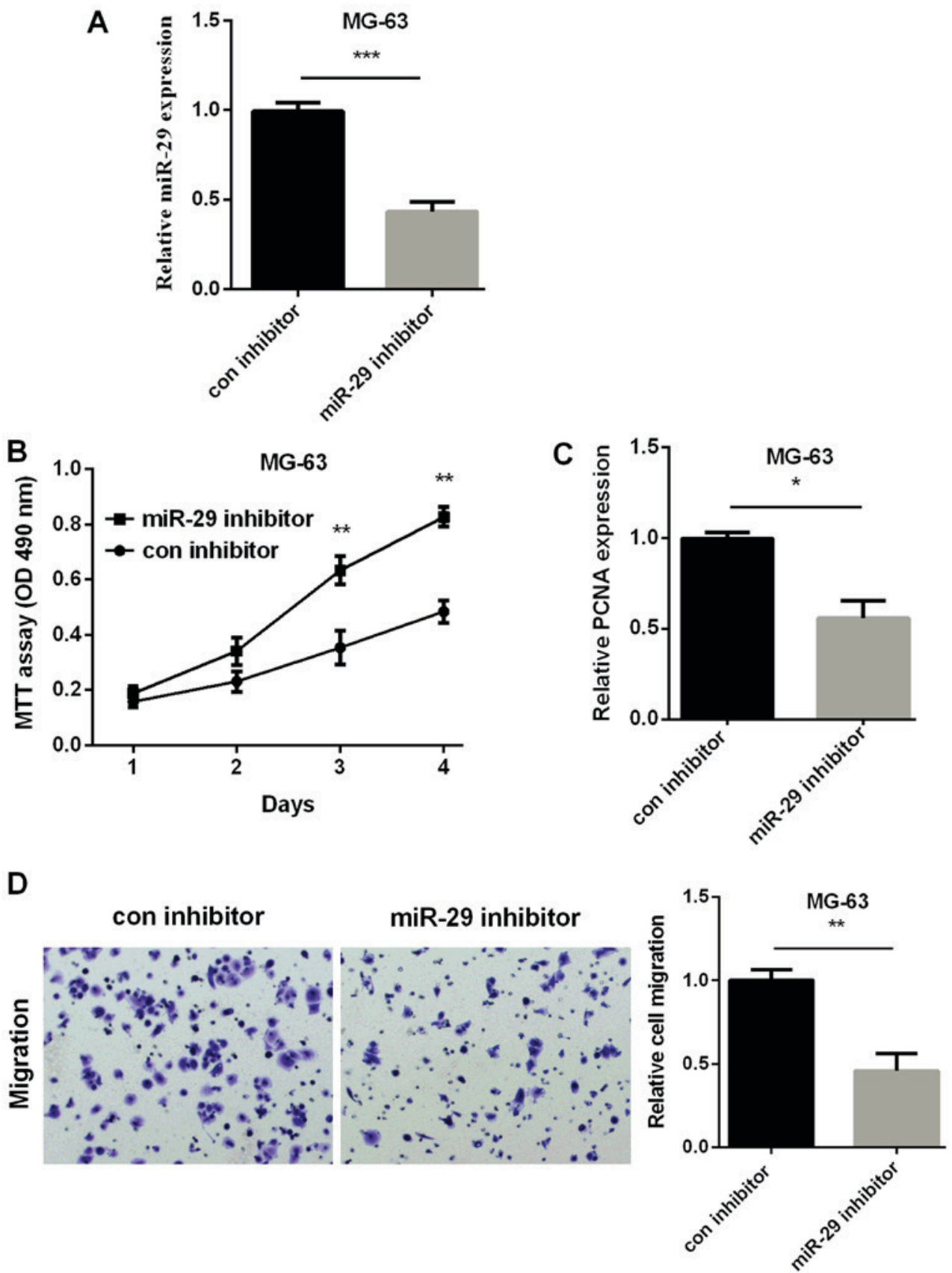

Figure 3. miR-29 inhibitor curbed OS cell migration and proliferation. (A) Quantification of miR-29 mRNA and protein level in MG-63 cells treated with miR-29 inhibitor. (B and C) Detection of cell viability by MTT and PCNA expression by RT-qPCR after MG-63 cells silencing miR-29. (D) Measurement of cell migration by Transwell after MG-63 cell silencing by miR-29. ${ }^{*} \mathrm{P}<0.05,{ }^{* * *} \mathrm{P}<0.01,{ }^{* * *} \mathrm{P}<0.001$. OS, osteosarcoma; PCNA, proliferating cell nuclear antigen.

\section{Results}

miR-29 is overexpressed in OS. Our primary aim was to explore miR-29 role in OS. First, we detected miR-29 level in OS tissues and cells using RT-qPCR. As shown in Fig. 1A, miR-29 average level in OS tissues was significantly increased compared with normal tissues. miR-29 expression in OS cells (MG-63, U2OS, 143B, Saos-2) was also elevated compared to normal cells (hFOB1.19), as expected (Fig. 1B). miR-29 expression was also detected in various stages of OS tumors and the results showed that the more advanced the tumor stage, the higher the expression of miR-29 (Fig. 1C), indicating that miR-29 may participate in OS malignant progression.
miR-29 mimic promotes OS cell proliferation and migration. Secondly, we measured miR-29 effect on OS progression. We overexpressed miR-29 in MG-63 cells using miR-29 mimic, and mRNA expression was increased significantly (Fig. 2A). Cell proliferation was measured by MTT assay and RT-qPCR. MTT results showed that cell viability in miR-29 mimic group was enhanced in comparison with control mimic group (Fig. 2B). PCNA is a marker for cell proliferation $(30,31)$. RT-qPCR analysis found that PCNA expression was increased in miR-29 mimic group (Fig. 2C). Cell migration measured by Transwell demonstrated that miR-29 mimic promoted OS cell migratory ability (Fig. 2D). The results above demonstrated a promotion effect of miR-29 on OS progression. 
A

\begin{tabular}{|c|c|c|}
\hline $\begin{array}{l}\text { Position } 676-683 \text { of PTEN } 3 \text { ' UTR } \\
\text { hsa-miR-29a-3p }\end{array}$ & $\begin{array}{l}5, \\
3^{\prime}\end{array}$ & 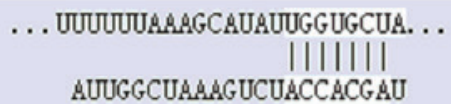 \\
\hline
\end{tabular}

B
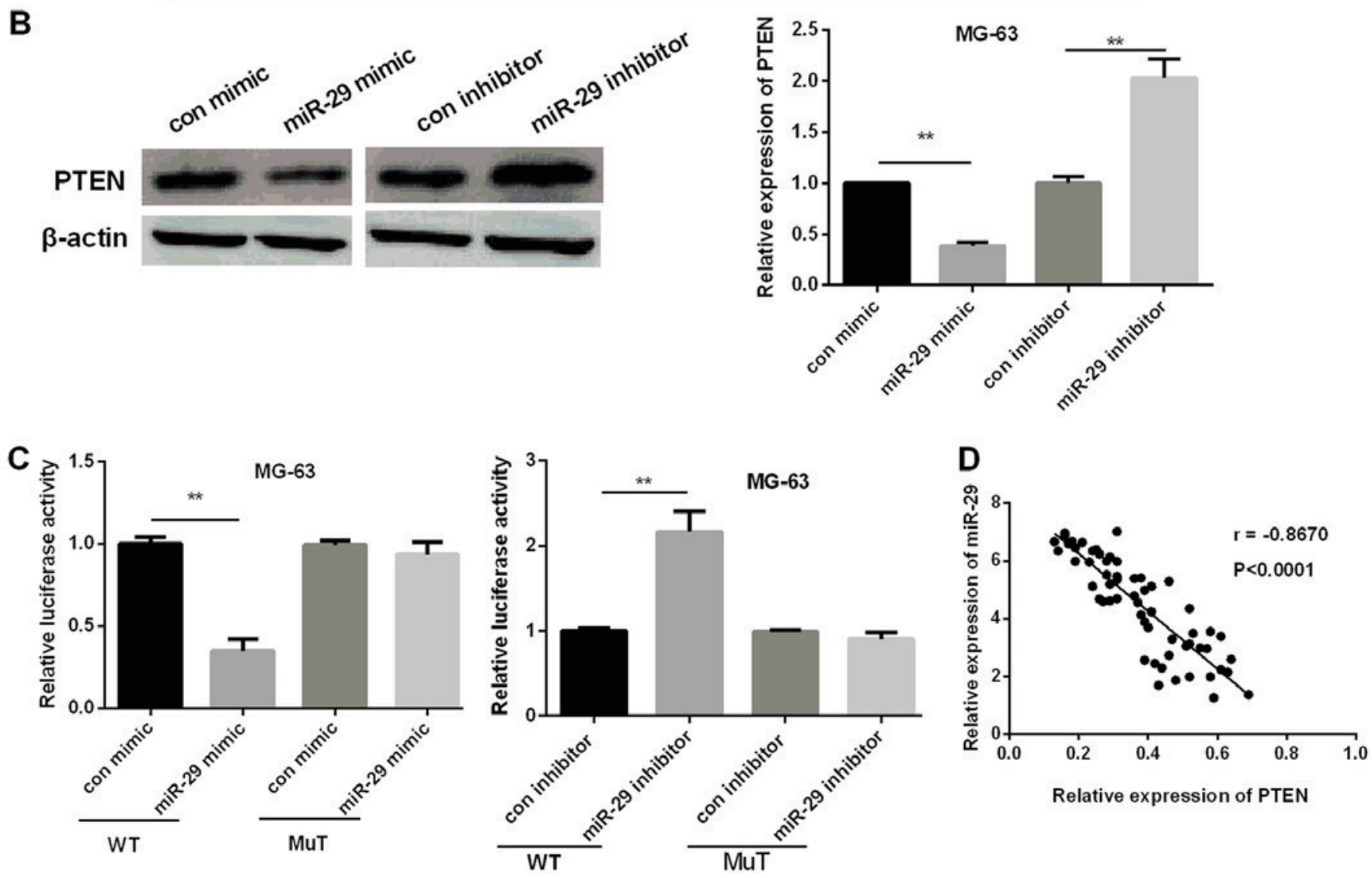

Figure 4. PTEN is a target of miR-29 in OS. (A) Binding sites of PTEN and miR-29. (B) PTEN protein and mRNA expression detected in MG-63 cells after restoration or silencing miR-29. (C) Detection of PTEN luciferase activity in MG-63 cells after beingtreated with PTEN 3'-UTR WT or MUT and miR-29 mimic. (D) Detection of the relevance of miR-29 and PTEN expression $(\mathrm{r}=-0.8670, \mathrm{P}<0.0001)$. ${ }^{* *} \mathrm{P}<0.01$. OS, osteosarcoma; PTEN, phosphatase and tensin homolog.

Silencing miR-29 curbed OS cell proliferation and migration. We then silenced miR-29 using miR-29 inhibitor to further confirm its effect on OS. The transfection efficiency is shown in Fig. 3A, miR-29 inhibitor group decreased miR-29 expression. As we expected, MTT results showed the decreased cell viability in miR-29 inhibitor group in comparison with the con-inhibitor group (Fig. 3B). RT-qPCR analysis revealed the increased PCNA expression in MG-63 cells after silencing miR-29 (Fig. 3C). Cell migration measured by Transwell showed enhanced cell migratory ability in miR-29 inhibitor group than the con-inhibitor group (Fig. 3D). The results above indicated that silencing miR-29 inhibited the development of OS.

miR-29 targets PTEN in OS. The next step was to investigate miR-29 molecular mechanism in OS. TargetScan Human 7.1 was carried out to identify the possible targets of miR-29 and results are shown in Fig. 4A. As further confirmation, we assessed PTEN protein and mRNA level after MG-63 cell restoration or inhibiting miR-29 via western blot analysis and RT-qPCR. PTEN expression was reduced when miR-29 was restored while increased when inhibiting miR-29 (Fig. 4B). Then, luciferase reporter assay was carried out to determine whether PTEN was a direct target of miR-29 in OS. The luciferase activity of miR-29 mimic group was decreased but increased in miR-29 inhibitor group in wild-type. However, there was no significant effect in mut type (Fig. 4C). The results verified PTEN as a direct target of miR-29. In addition, miR-29 and PTEN expression were negatively correlated (Fig. 4D).

PTEN attenuates miR-29 promotion effect on OS progression. Then, we investigated the role of PTEN in OS and whether PTEN affected the miR-29 role in regulating OS progression. PTEN was overexpressed by PTEN vector due to its lower expression in OS. The results showed that PTEN expression was higher after cells were transfected with PTEN vector (Fig. 5A). PTEN overexpressed could inhibit OS cell migration and proliferation (Fig. 5B and C). Subsequently, miR-29 mimic and PTEN vector were co-transfected into MG-63 cells to test the role of PTEN in OS regulated by miR-29. The cell viability and PCNA expression were both increased obviously by miR-29 mimic in comparison with control (Fig. 5D). By contrast, overexpression of both miR-29 and PTEN, cell proliferation was reduced compared with overexpression of miR-29 alone, indicating that PTEN could attenuate miR-29 promotion effect on cell proliferation. Additionally, the migration results suggested that miR-29 promotion increased migration in OS cells compared with control, whereas promoting both 

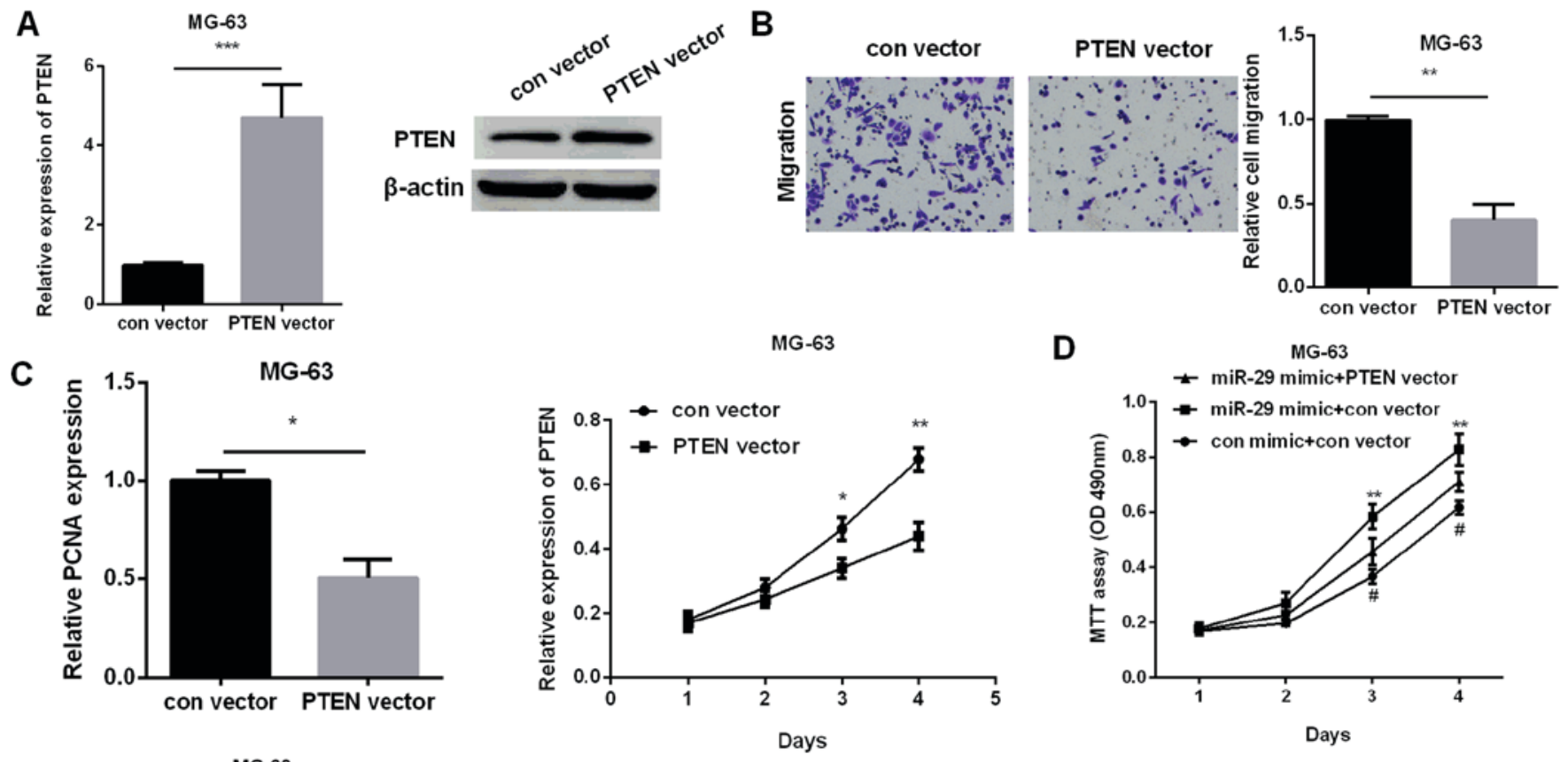

D
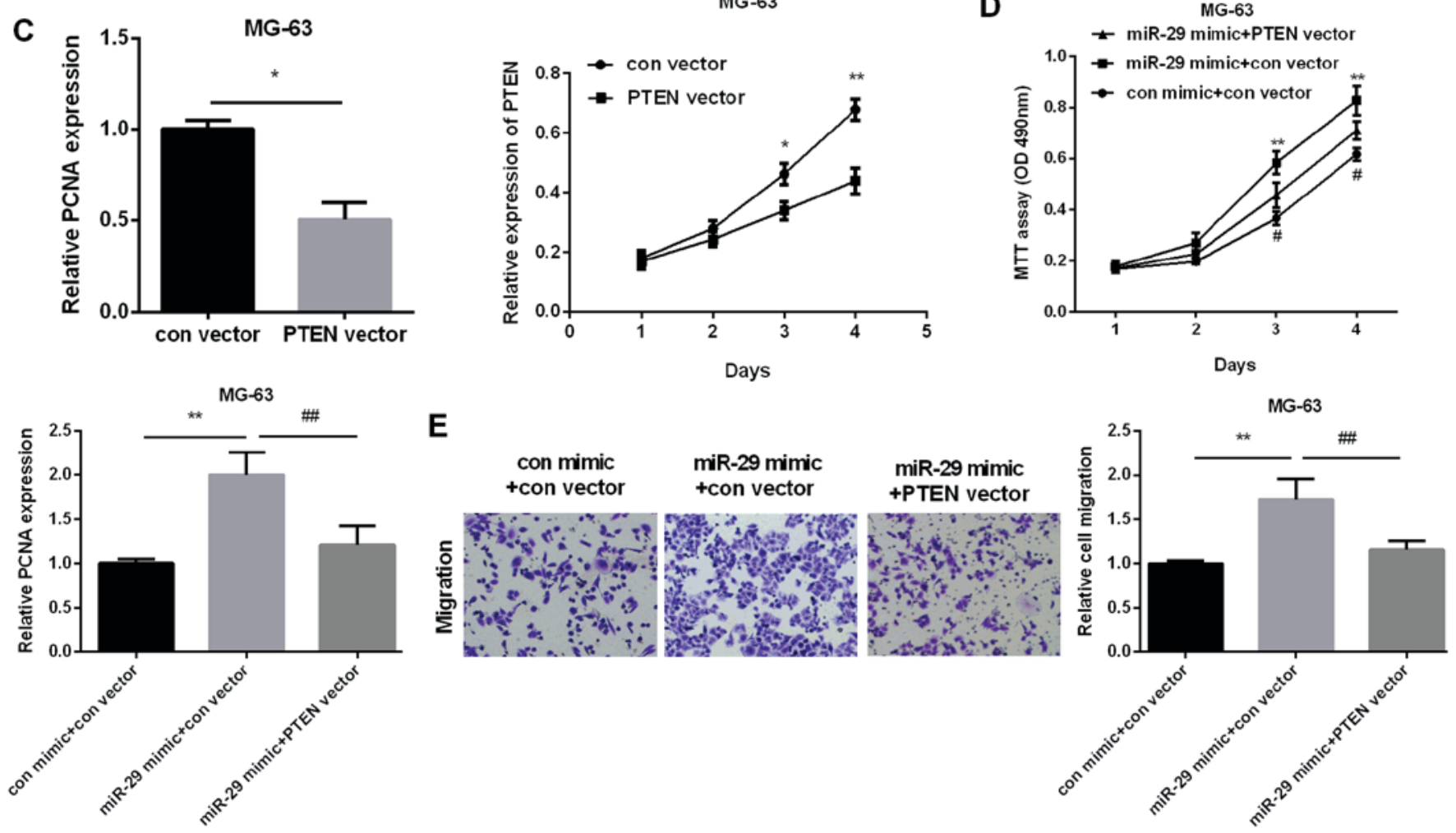

Figure 5. PTEN attenuates miR-29 promotion effect on OS cell migration and proliferation. (A) PTEN mRNA and protein expression quantified in MG-63 cells after overexpression of PTEN. (B) Measurement of cell migration by Transwell after MG-63 cell overexpression of PTEN. (C) Detection of cell viability by MTT and PCNA expression using RT-qPCR after MG-63 cells after overexpression of PTEN. (D) Detection of cell viability by MTT and PCNA expression by RT-qPCR after MG-63 cell re-expression of miR-29 alone or both miR-29 and PTEN. (E) Detection of cell migration by Transwell after MG-63 cell re-expression of miR-29 alone or both miR-29 and PTEN. ${ }^{*} \mathrm{P}<0.05,{ }^{* * *} \mathrm{P}<0.01,{ }^{* * *} \mathrm{P}<0.001 ;{ }^{*} \mathrm{P}<0.05,{ }^{\# \#} \mathrm{P}<0.01$. OS, osteosarcoma; PTEN, phosphatase and tensin homolog; PCNA, proliferating cell nuclear antigen.

miR-29 and PTEN reduced cell migration in comparison with promoting miR-127 alone, suggesting that PTEN could reverse miR-29 promotion effect on OS cell migration. In summary, miR-29 could facilitate OS cell migration and proliferation by targeting PTEN.

\section{Discussion}

An increasing number of studies have reported the important roles of miRNAs in tumorigenesis and tumor development (32). Furthermore, the ability to control cell growth, metastasis and survival may serve essential roles in preventing and treating various human malignancies, including OS (33). miR-29 was reported to have significant inhibitory effect on various cancers. For example, it inhibited hepatocellular carcinoma cell migration and proliferation by regulating IGF1R (34). miR-29 played a tumor suppressive role in modulating gliomas through targeting CDC42 (35). However, Gao et al reported that miR-29 played a promoting role in OS (36) and Hong et al showed the same discovery that miR-29 expression was higher in OS patients (37). Those results were in line with our findings that miR-29 expression was upregulated in OS tissues and cells, but these studies did not show the function of miR-29 in OS tumorigenesis and development. In the present study, we first demonstrated that restoration of miR-29 facilitated cell migration and proliferation, while inhibiting miR-29 showed inhibitory effect.

PTEN was the first tumor suppressor gene found with a bispecific phosphatase activity and closely associated with carcinoma (38). PTEN suppressed the migration and genetic deletion of the PTEN tumor suppressor gene, promoting cell motility and overexpression or reconstitution of PTEN and inhibiting cell motility in a variety of cell types $(39,40)$. Hundreds of genes were found to be the target of miRNA, which harbors the target sequence in their 3'-UTR complementary to the seed region of the miRNA. PTEN was also proven to be a target of miRNAs in modulating cancer progression. PTEN was the target of miR-28 and suppressed gastric cancer 
cell proliferation and invasion (41). Additionally, it suppressed renal cancer cell proliferation as a target of miR-30a (42). Zhao et al reported that PTEN regulated OS cell growth and apoptosis and acted as a target of miR-19 (43). We also found that the PTEN mRNA expression was downregulated in MG63 cells compared with the hFOB1.19 cells (data not shown). However, the underlying mechanism between miR-29 and PTEN was still unclear in OS. In our study, we found that miR-29 could negatively regulate the expression of PTEN in MG63 cells. Further investigation was conducted to explore the molecular mechanism how PTEN promoted OS cell proliferation and migration. In addition, PTEN overexpressed was proved to inhibit OS cell proliferation and migration, PTEN could reverse miR-29 promotion effect on OS progression as well. Therefore, to the best of our knowledge, the present study provided an effective treatment target in OS.

In conclusion, miR-29 facilitated OS migration and proliferation and this is the first report that miR-29 targeted PTEN and regulated OS development. Furthermore, PTEN could reverse miR-29 promotion effect on OS providing a new idea for OS therapy.

\section{Acknowledgements}

Not applicable.

\section{Funding}

This study received the specific grant from the Co-construction project of Henan Provincial Medical Science and Technology Key Project (grant no. 201401007).

\section{Availability of data and materials}

The datasets generated or analyzed during the present study are available from the corresponding author on reasonable request.

\section{Authors' contributions}

QL was a major contributor in writing the manuscript and contributed to the conception of the study. PG contributed significantly to data analysis and manuscript preparation. LS performed the data analyses and wrote the manuscript. QW and PW helped perform the data analysis with constructive discussions. All authors read and approved the final study.

\section{Ethics approval and consent to participate}

The study was approved by the Ethics Committee of The First Affiliated Hospital of Zhengzhou University (Zhengzhou, China). Signed informed consents were obtained from all participants.

\section{Patient consent for publication}

Not applicable.

\section{Competing interests}

The authors declare that they have no competing interests.

\section{References}

1. Endo-Munoz L, Evdokiou A and Saunders NA: The role of osteoclasts and tumour-associated macrophages in osteosarcoma metastasis. Biochim Biophys Acta 1826: 434-442, 2012.

2. Harting MT and Blakely ML: Management of osteosarcoma pulmonary metastases. Semin Pediatr Surg 15: 25-29, 2006.

3. Jia S and Li B: Osteosarcoma of the jaws: Case report on synchronous multicentric osteosarcomas. J Clin Diagn Res 8: ZD01-ZD03, 2014.

4. He JP, Hao Y, Wang XL, Yang XJ, Shao JF, Guo FJ and Feng JX: Review of the molecular pathogenesis of osteosarcoma. Asian Pac J Cancer Prev 15: 5967-5976, 2014.

5. Egas-Bejar D, Anderson PM, Agarwal R, Corrales-Medina F, Devarajan E, Huh WW, Brown RE and Subbiah V: Theranostic profiling for actionable aberrations in advanced high risk osteosarcoma with aggressive biology reveals high molecular diversity: The human fingerprint hypothesis. Oncoscience 1: 167-179, 2014.

6. Poletajew S, Fus L and Wasiutyński A: Current concepts on pathogenesis and biology of metastatic osteosarcoma tumors. Ortop Traumatol Rehabil 13: 537-545, 2011.

7. Xu C, Ping Y, Li X, Zhao H, Wang L, Fan H, Xiao Y and Li X: Prioritizing candidate disease miRNAs by integrating phenotype associations of multiple diseases with matched miRNA and mRNA expression profiles. Mol Biosyst 10: 2800-2809, 2014

8. Gaál Z and Oláh E: MicroRNA-s and their role in malignant hematologic diseases. Orv Hetil 153: 2051-2059, 2012 (In Hungarian).

9. Lin S, Pan L, Guo S, Wu J, Jin L, Wang JC and Wang S: Prognostic role of microRNA-181a/b in hematological malignancies: A meta-analysis. PLoS One 8: e59532, 2013.

10. Kalinowski FC, Brown RA, Ganda C, Giles KM, Epis MR, Horsham J and Leedman PJ: microRNA-7: A tumor suppressor miRNA with therapeutic potential. Int J Biochem Cell Biol 54: 312-317, 2014.

11. Xu Y, Chu H, Zhou Y, Wang J, Dong C and Yin R: miR-365 functions as a tumor suppressor by directly targeting CYR61 in osteosarcoma. Biomed Pharmacother 98: 531-537, 2018.

12. Yu L, Wang S, Lin X, Lu Y and Gu P: MicroRNA-124a inhibits cell proliferation and migration in liver cancer by regulating interleukin-11. Mol Med Rep 17: 3972-3978, 2018.

13. Luo Z, Wu G, Zhang D, Liu J and Ran R: microRNA 625 targets Yes associated protein 1 to suppress cell proliferation and invasion of osteosarcoma. Mol Med Rep 17: 2005-2011, 2018

14. Wang N, Li P, Liu W, Wang N, Lu Z, Feng J, Zeng X, Yang J, Wang $\mathrm{Y}$ and Zhao W: miR-141-3p suppresses proliferation and promotes apoptosis by targeting GLI2 in osteosarcoma cells. Oncol Rep 39: 747-754, 2018.

15. He Y and Yu B: MicroRNA-93 promotes cell proliferation by directly targeting P21 in osteosarcoma cells. Exp Ther Med 13: 2003-2011, 2017.

16. Sun Z, Liu Q, Hong H, Zhang H and Zhang T: miR-19 promotes osteosarcoma progression by targeting SOCS6. Biochem Biophys Res Commun 495: 1363-1369, 2018.

17. Cai Q, Zeng S, Dai X, Wu J and Ma W: miR-504 promotes tumour growth and metastasis in human osteosarcoma by targeting TP53INP1. Oncol Rep 38: 2993-3000, 2017.

18. Wu Z, Huang X, Huang X, Zou Q and Guo Y: The inhibitory role of Mir-29 in growth of breast cancer cells. J Exp Clin Cancer Res 32: 98, 2013.

19. Lv M, Zhong Z, Huang M, Tian Q, Jiang R and Chen J: lncRNA H19 regulates epithelial-mesenchymal transition and metastasis of bladder cancer by miR-29b-3p as competing endogenous RNA. Biochim Biophys Acta 1864: 1887-1899, 2017.

20. Kwon JJ, Willy JA, Quirin KA, Wek RC, Korc M, Yin XM and Kota J: Novel role of miR-29a in pancreatic cancer autophagy and its therapeutic potential. Oncotarget 7: 71635-71650, 2016.

21. Shin J, Shim HG, Hwang T, Kim H, Kang SH, Dho YS, Park SH, Kim SJ and Park CK: Restoration of miR-29b exerts anti-cancer effects on glioblastoma. Cancer Cell Int 17: 104, 2017.

22. Ma J, Li T, Yuan H, Han X, Shui S, Guo D and Yan L: MicroRNA-29a inhibits proliferation and motility of schwannoma cells by targeting CDK6. J Cell Biochem 119: 2617-2626, 2018.

23. Ding Q, Chang CJ, Xie X, Xia W, Yang JY, Wang SC, Wang Y, Xia J, Chen L, Cai C, et al: APOBEC3G promotes liver metastasis in an orthotopic mouse model of colorectal cancer and predicts human hepatic metastasis. J Clin Invest 121: 4526-4536, 2011. 
24. Fabbri M, Garzon R, Cimmino A, Liu Z, Zanesi N, Callegari E, Liu S, Alder H, Costinean S, Fernandez-Cymering C, et al: MicroRNA-29 family reverts aberrant methylation in lung cancer by targeting DNA methyltransferases $3 \mathrm{~A}$ and 3B. Proc Natl Acad Sci USA 104: 15805-15810, 2007.

25. Boosani CS and Agrawal DK: PTEN modulators: A patent review. Expert Opin Ther Pat 23: 569-580, 2013.

26. Gao ZQ, Wang JF, Chen DH, Ma XS, Wu Y, Tang Z and Dang XW: Long non-coding RNA GAS5 suppresses pancreatic cancer metastasis through modulating miR-32-5p/PTEN axis. Cell Biosci 7: 66, 2017.

27. Wang P, Guan Q, Zhou D, Yu Z, Song Y and Qiu W: miR-21 inhibitors modulate biological functions of gastric cancer cells via PTEN/PI3K/mTOR pathway. DNA Cell Biol 37: 38-45, 2018

28. Guo J, Xiao Z, Yu X and Cao R: miR-20b promotes cellular proliferation and migration by directly regulating phosphatase and tensin homolog in prostate cancer. Oncol Lett 14: 6895-6900, 2017.

29. Livak KJ and Schmittgen TD: Analysis of relative gene expression data using real-time quantitative PCR and the 2(-Delta Delta C(T)) method. Methods 25: 402-408, 2001.

30. Hall PA, Levison DA, Woods AL, Yu CC, Kellock DB, Watkins JA, Barnes DM, Gillett CE, Camplejohn R, Dover R, et al: Proliferating cell nuclear antigen (PCNA) immunolocalization in paraffin sections: An index of cell proliferation with evidence of deregulated expression in some neoplasms. J Pathol 162: 285-294, 1990.

31. Moldovan GL, Pfander B and Jentsch S: PCNA, the maestro of the replication fork. Cell 129: 665-679, 2007.

32. Li Y, Zeng C, Tu M, Jiang W, Dai Z, Hu Y, Deng Z and Xiao W: MicroRNA-200b acts as a tumor suppressor in osteosarcoma via targeting ZEB1. Onco Targets Ther 9: 3101-3111, 2016.

33. Yin Z, Ding H, He E, Chen J and Li M: Up-regulation of microRNA-491-5p suppresses cell proliferation and promotes apoptosis by targeting FOXP4 in human osteosarcoma. Cell Prolif 50: e12308, 2017.

34. Wang X, Liu S, Cao L, Zhang T, Yue D, Wang L, Ping Y, He Q, Zhang C, Wang M, et al: miR-29a-3p suppresses cell proliferation and migration by downregulating IGF1R in hepatocellular carcinoma. Oncotarget 8: 86592-86603, 2017.
35. Shi C, Ren L, Sun C, Yu L, Bian X, Zhou X, Wen Y, Hua D, Zhao S, Luo W, et al: miR-29a/b/c function as invasion suppressors for gliomas by targeting CDC42 and predict the prognosis of patients. Br J Cancer 117: 1036-1047, 2017.

36. Gao S, Cheng C, Chen H, Li M, Liu K and Wang G: IGF1 3'UTR functions as a ceRNA in promoting angiogenesis by sponging miR-29 family in osteosarcoma. J Mol Histol 47: 135-143, 2016.

37. Hong Q, Fang J, Pang Y and Zheng J: Prognostic value of the microRNA-29 family in patients with primary osteosarcomas. Med Oncol 31: 37, 2014

38. Li J, Yen C, Liaw D, Podsypanina K, Bose S, Wang SI, Puc J, Miliaresis C, Rodgers L, McCombie R, et al: PTEN, a putative protein tyrosine phosphatase gene mutated in human brain, breast, and prostate cancer. Science 275: 1943-1947, 1997.

39. Yang YK, XiWY,Xi RX,Li JY,Li Q and Gao YE: MicroRNA-494 promotes cervical cancer proliferation through the regulation of PTEN. Oncol Rep 33: 2393-2401, 2015.

40. Hodakoski C, Fine B, Hopkins B and Parsons R: Analysis of intracellular PTEN signaling and secretion. Methods 77-78: 164-171, 2015.

41. Li L, Zhu X, Shou T, Yang L, Cheng X, Wang J, Deng L and Zheng Y: MicroRNA-28 promotes cell proliferation and invasion in gastric cancer via the PTEN/PI3K/AKT signalling pathway. Mol Med Rep 17: 4003-4010, 2018.

42. Li J, Li C, Li H, Zhang T, Hao X, Chang J and Xu Y: MicroRNA 30 a $5 p$ suppresses tumor cell proliferation of human renal cancer via the MTDH/PTEN/AKT pathway. Int J Mol Med 41: 1021-1029, 2018

43. Zhao D, Chen Y, Chen S, Zheng C, Hu J and Luo S: MiR-19a regulates the cell growth and apoptosis of osteosarcoma stem cells by targeting PTEN. Tumour Biol 39: 1010428317705341, 2017.

This work is licensed under a Creative Commons Attribution-NonCommercial-NoDerivatives 4.0 International (CC BY-NC-ND 4.0) License. 